\title{
Article
}

\section{Evaporative Organization of Hierarchically Structured Polymer Blend Rings} Myunghwan Byun, Suck Won Hong, Feng Qiu, Qingze Zou, and Zhiqun Lin Macromolecules, 2008, 41 (23), 9312-9317 • Publication Date (Web): 07 November 2008 Downloaded from http://pubs.acs.org on December 2, 2008
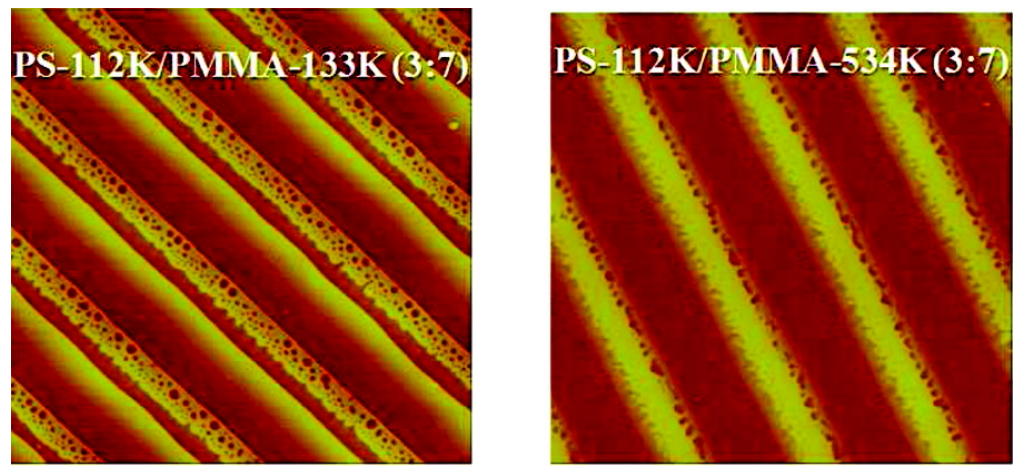

\section{More About This Article}

Additional resources and features associated with this article are available within the HTML version:

- Supporting Information

- Access to high resolution figures

- $\quad$ Links to articles and content related to this article

- Copyright permission to reproduce figures and/or text from this article

\section{View the Full Text HTML}




\title{
Evaporative Organization of Hierarchically Structured Polymer Blend Rings
}

\author{
Myunghwan Byun, ${ }^{\dagger}$ Suck Won Hong, ${ }^{\dagger}$ Feng Qiu, ${ }^{*}$ Qingze Zou, ${ }^{\S}$ and Zhiqun Lin*, ${ }^{\dagger}$ \\ Department of Materials Science and Engineering, Iowa State University, Ames, Iowa 50011; \\ Department of Macromolecular Science and the Key Laboratory of Molecular Engineering of Polymers \\ at Fudan University, Shanghai, China 200433; and Department of Mechanical Engineering, Iowa State \\ University, Ames, Iowa 50011
}

Received August 15, 2008; Revised Manuscript Received October 4, 2008

\begin{abstract}
We report the first study of the controlled, evaporative self-organization of a polymer blend from a restricted geometry comprised of a spherical lens upon a Si substrate (i.e., a sphere-on-flat geometry). This geometry facilitated the control over the evaporation rate of solvent, thereby eliminating the temperature gradient and the possible convective instabilities. In this study, a drop of polystyrene (PS) and poly(methyl methacrylate) (PMMA) toluene solution evaporated in the sphere-on-flat geometry. The combination of controlled, consecutive pinning-depinning cycles (i.e., "stick-slip") of the contact line at the edge of the geometry, spontaneous phase separation of incompatible polymers at the microscopic scale, and a dewetting process in the late stage of phase segregation led to the formation of gradient, hierarchically structured polymer blend rings composed of phaseseparated PS and PMMA. The topographic distribution of PS and PMMA phases on the ring surface were revealed after removal of the PS phase with a selective solvent. Namely, the trench-pit structures were formed in the PS-112K/PMMA-133K blend, while for the PS-112K/PMMA-534K blend, pit morphologies were observed. This facile approach offers a new way of simultaneously processing two or more nonvolatile components via controlled evaporation to produce new kinds of structures with hierarchical order in a simple, robust, and one-step manner.
\end{abstract}

\section{Introduction}

Self-organization of polymers and nanocrystals at the solution/ air interface driven by evaporation produces a variety of complex, dissipative, and far-from-equilibrium patterns including "coffee rings" $1-3$ and polygonal network structures ${ }^{4-7}$ that generally possess a low degree of regularity and long-range order. It would be of great interest and technological importance to explore the full potential of the evaporation process for assembling ordered micro- and nanostructures for applications in electronics, optics, optoelectronics, and sensory devices. ${ }^{8-10}$ To date, a few elegant studies have centered on establishing a means of harnessing the drying process of an evaporating droplet to yield highly regular structures. For example, well-aligned stripe patterns of nanoparticles were obtained through dewetting of a dilute film of polymer-coated nanoparticles flowing on a water surface using a programmable dip-coating process. ${ }^{11}$ Rather than allowing a droplet to freely evaporate, regular polymer patterns have been produced continuously from a receding meniscus, with the polymer solution confined between two parallel plates, by controlling the speed of the upper sliding plate while keeping the lower plate stationary. ${ }^{12}$ Recently, concentric "coffee rings" of polymers (or nanocrystals) of high fidelity and regularity have been obtained by confining a drop of polymer (or nanocrystals) solution in a restricted geometry composed of a spherical lens on a flat substrate (i.e., sphereon-flat geometry). ${ }^{13-22}$ Because of imposed geometrical constrain, the evaporation was constrained to occur only at the droplet edges, ${ }^{13,14}$ which in turn led to the formation of highly ordered concentric rings. ${ }^{13,14}$ It is noteworthy that these previous studies have solely centered on single nonvolatile solute system (e.g., homopolymers, colloidal particles, or nanocrystals). By contrast, structure formation from evaporating droplets consist-

\footnotetext{
* To whom correspondence should be addressed. E-mail: zqlin@iastate.edu. sity.

† Department of Materials Science and Engineering, Iowa State Univer-

Fudan University.

${ }^{\S}$ Department of Mechanical Engineering, Iowa State University.
}

ing of two or more nonvolatile solutes, e.g., binary polymer blend, has been less explored regardless of its common existence and numerous technological implication. The synergy of phase separation of polymer blends, and their destabilization mediated by the interaction between solutes and substrates during controlled solvent evaporation, can lead to the formation of ordered complex structures.

Phase separation phenomena play a key role in the pattern evolution of a binary mixture of liquid composed of various condensed matter including polymers, surfactants, colloids, emulsions, and biological materials. The phase separation kinetics of polymer blend has been studied extensively to understand the phase demixing mechanism ${ }^{23-26}$ in conjunction with dewetting ${ }^{27-31}$ and explore the characteristic parameters that influence the phase separation. ${ }^{32-34}$ Recently, the creation of multiscale stripelike patterns in thin polymer blend films was demonstrated; the patterns resulted from the interplay of evaporation, convective flow, differential diffusion, and spinodal decomposition. ${ }^{35}$

Herein, we report our first study on the spontaneous formation of hierarchically structured polymer blend patterns via controlled, evaporative self-organization of an incompatible, binary polymer blend solution in a sphere-on-flat geometry. PS and PMMA were selected as nonvolatile solutes. The topographic distribution of PS and PMMA phases on the pattern surface were revealed after removal of the PS phase with a selective solvent. Distinct difference in the surface morphology was observed for the polymer blends with different molecular weight of PMMA. Self-organized trench-pit structures were formed in the PS-112K/PMMA-133K blend, while pit morphologies were observed in the PS-112K/PMMA-534K blend. This facile approach offers a new way of simultaneously processing two or more nonvolatile components via controlled evaporation to produce new kinds of structures with hierarchical order in a simple, robust, and one-step manner. 




Figure 1. (a) Three-dimensional illustration of a drop of PS/PMMA toluene solution trapped between a spherical lens and a Si substrate (i.e., a sphere-on-flat geometry). (b) Cross section of the capillaryheld PS/PMMA solution in (a). The concentric rings were produced by controlled, repetitive "stick-slip" motion of the contact line. The distance of the rings away from the sphere/Si contact center is $X_{n}(n=$ $1-3 ; X_{1}, X_{2}$, and $X_{3}$ correspond to the outmost, intermediate, and innermost region, respectively, where the rings were formed).

\section{Experimental Section}

Materials and Sample Preparation. Polystyrene (PS; numberaverage molecular weight, $M_{\mathrm{n}}=112 \mathrm{~kg} / \mathrm{mol}$, polydispersity index, $\mathrm{PDI}=1.05)$ and poly(methyl methacrylate) (PMMA; $M_{\mathrm{n}}=133$ $\mathrm{kg} / \mathrm{mol}$, PDI $=1.64$ and $M_{\mathrm{n}}=534 \mathrm{~kg} / \mathrm{mol}$, PDI $=1.57$ ) were selected as nonvolatile solutes to prepare the PS/PMMA blends due to their strong immiscibility. Two polymer blends, namely, PS-112K/PMMA-133K and PS-112K/PMMA-534K, were used in the study. The weight ratio of PS to PMMA is 3:7, i.e., forming an asymmetric binary mixture. PS and PMMA were dissolved in toluene to prepare the PS/PMMA toluene solution at concentration, $c=0.5 \mathrm{mg} / \mathrm{mL}$. Subsequently, the solutions were purified with $0.2 \mu \mathrm{m}$ hydrophobic membrane filters.

Restricted Geometry. To construct a restricted geometry (i.e., sphere-on-flat geometry), a spherical lens and a Si wafer were used. The spherical lens made from fused silica with a radius of curvature, $R \sim 2.0 \mathrm{~cm}$, and $\mathrm{Si}$ substrates were cleaned by a mixture of sulfuric acid and Nonchromix. Subsequently, they were rinsed with DI water and blow-dried with $\mathrm{N}_{2}$. The sphere and $\mathrm{Si}$ were firmly fixed at the top and bottom of sample holders inside a sealed chamber, respectively. To implement a restricted geometry, an inchworm motor with a step motion of a few micrometers was used to place the upper sphere into contact with the lower stationary Si surface. Before contact, with just a few hundred micrometers between the surfaces, a drop of $20 \mu \mathrm{L}$ of $05 \mathrm{mg} / \mathrm{mL}$ PS/PMMA toluene solution was loaded and trapped within the gap between the sphere and $\mathrm{Si}$ due to the capillary force. The sphere was finally brought into contact with the Si substrate by the inchworm motor such that a capillary-held PS/PMMA blend solution was formed with evaporation rate highest at the edge of the capillary, as seen in Figure 1.

Characterization. The evaporation took less than $1 \mathrm{~h}$ to complete. Afterward, the sphere and the Si substrate were separated. Because of the curvature effect of the sphere, only the patterns formed on the Si substrate were evaluated by the optical microscope (OM; Olympus BX51 in reflection mode) and atomic force microscopy (AFM; Dimension 3100 scanning force microscope in tapping mode (Digital Instruments)). BS-tap300 tips (Budget Sensors) with spring constants ranging from 20 to $75 \mathrm{~N} / \mathrm{m}$ were used as scanning probes. Prior to the OM and AFM measurements, a scratch on the sample was made so that we were able to compare the surface morphologies at the same location before and after removal PS of with a selective solvent, cyclohexane.

\section{Results and Discussion}

The PS/PMMA toluene solution was confined in the sphereon-flat geometry (i.e., forming a capillary-held solution); thus, the evaporation of toluene can only occur at the edge of the capillary (Figure 1). Evaporative loss of toluene triggered the transportation of the PS/PMMA to the edge, forming an outmost PS/PMMA "coffee ring" (i.e., pinning of the contact line at the solution/air interface; "stick" step). ${ }^{2}$ During the deposition of the PS/PMMA ring, the initial contact angle of the capillary edge decreased gradually due to the evaporation of toluene to a critical angle, at which the capillary force (depinning force) became larger than the pinning force. ${ }^{14}$ This caused the contact line to jump to a new position (i.e., "slip" step), and a new ring was thus developed. Consecutive "stick-slip" cycles of the receding contact line toward the sphere/Si contact center with elapsed time led to the regular assemblies of PS/PMMA concentric rings governed by the competition between the capillary force and the pinning force. ${ }^{14}$ We note that in previous studies investigating the interfacial interaction of homopolymers, i.e., PS and PMMA, with Si substrate during the controlled evaporation, PS was found to form fingering instabilities along the microscopic rings, while PMMA produced well-organized concentric rings. ${ }^{18}$ Therefore, in the present study of controlled evaporative self-organization of the PS/PMMA blend, only the blend at the weight ratio of PS/PMMA $=3 / 7$ was used to avoid the fingering instabilities of PS, as seen, for example, in the PS-112K/PMMA-133K blends with $7 / 3$ and 5/5 weight ratios (see Figure 1S in Supporting Information).

Figure 2 shows representative optical micrographs of highly ordered, microscopic PS/PMMA concentric rings formed at the intermediate region, $X_{2}$, for the PS-112K/PMMA-133K blend (Figure 2a,b) and produced in between the intermediate region, $X_{2}$, and the innermost region, $X_{3}$, for the PS-112K/PMMA-534K blend (Figure 2c,d), where $X$ is the distance away from the sphere/Si contact center (Figure 1b). Comparison of parts a and b of Figure 2 revealed that the surface morphologies of the PS112K/PMMA-133K concentric rings were altered after removal of the PS-112K phase with cyclohexane, a selective solvent for PS and nonsolvent for PMMA, in that a thick dark curves appeared in the middle of the rings. With the other blend, PS112K/PMMA-534K, a different morphology after removing the PS-112K phase was observed; namely, the integrity of originally formed PS-112K/PMMA-534K rings was retained, and the apparent deposits of polymers in between the concentric rings were removed with cyclohexane (Figure 2c,d).

To scrutinize the surface morphologies of the PS/PMMA rings and the detailed domain structures of PS and PMMA within the ring and, more importantly, to understand their structure formation due to the phase separation during and/or after the solvent evaporation, AFM measurements were performed. 2D AFM height images of the PS-112K/PMMA-133K concentric rings before and after removal of the PS phase and the corresponding profiles scanning over a short distance are shown in Figure 3. Locally, they appeared as parallel stripes. Notably, the center-to-center distance between adjacent stripes, $\lambda_{\mathrm{C}-\mathrm{C}}$, the width of the stripe, $w$, and the height of the stripe, $h$, decreased with increased proximity to the sphere/Si contact center, from $\lambda_{\mathrm{C}-\mathrm{C}}=19 \mu \mathrm{m}, w=16 \mu \mathrm{m}$, and $h=200 \mathrm{~nm}$ at $X_{1}=3950 \mu \mathrm{m}$ (outmost region; Figure 3a) to $13 \mu \mathrm{m}, 11 \mu \mathrm{m}$, and $170 \mathrm{~nm}$ at $X_{2}=2980 \mu \mathrm{m}$ (intermediate region; Figure $3 \mathrm{e}$ ) to $11 \mu \mathrm{m}, 9$ $\mu \mathrm{m}$, and $130 \mathrm{~nm}$ at $X_{3}=2235 \mu \mathrm{m}$ (innermost region; Figure $3 i)$, where $X_{n}(n=1-3)$ was defined in Figure 1b. This observation can be attributed to the competition between linear pinning force and nonlinear capillary force. ${ }^{14}$ Line scan of the stripes revealed the dimplelike fluctuations of in the middle of the stripe (marked under red arrows in Figure 3b,f,j). After thorough washing of the ring patterns with cyclohexane, clear height images with detailed domain structures were obtained (Figure 3c,d,g,h,k,l). The depth of fluctuation dramatically increased, yielding concentric trenches in the rings (under red 

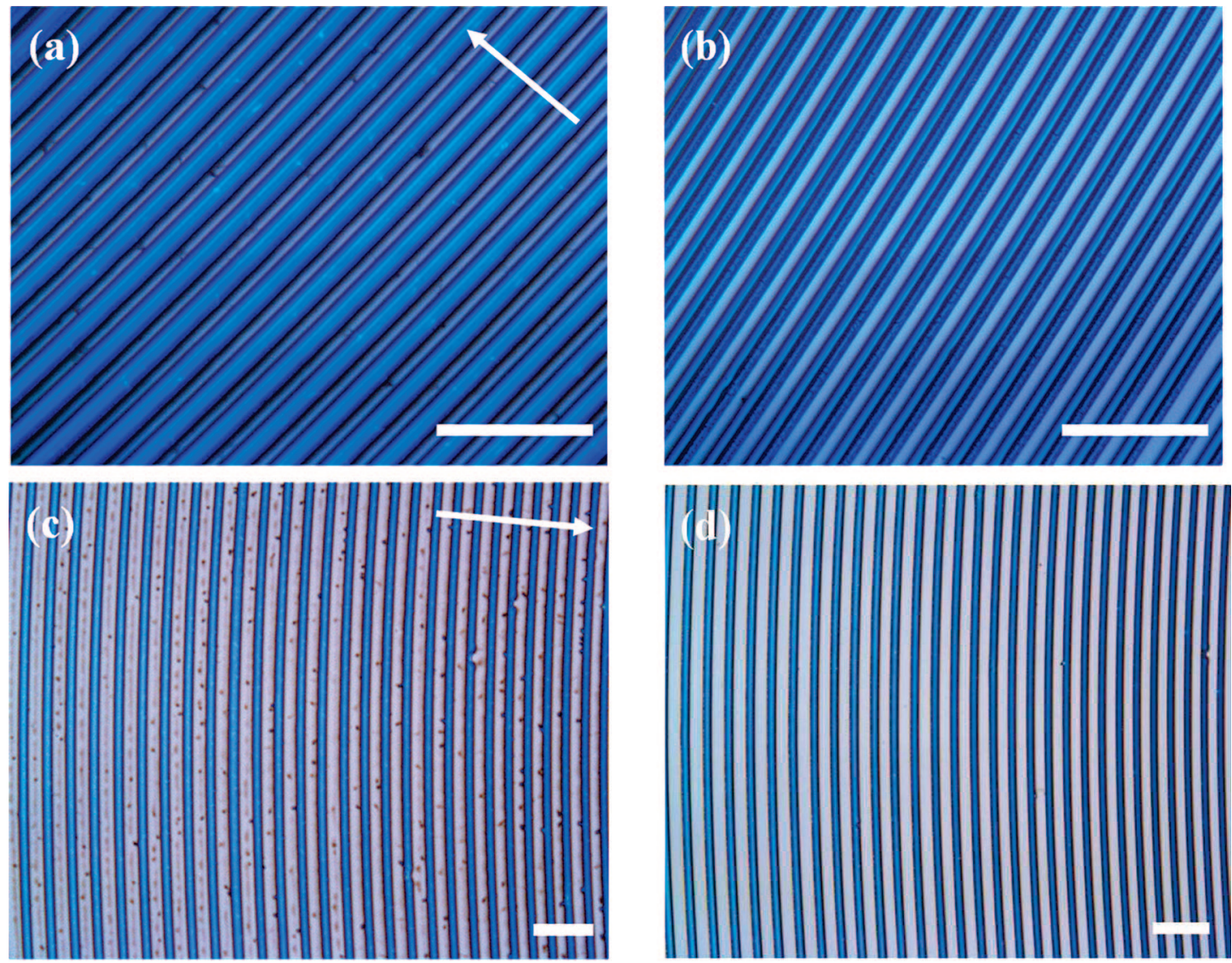

Figure 2. Optical micrographs of concentric rings in blue consisting of PS/PMMA blend formed by drying the PS/PMMA toluene solution $(c=$ $0.5 \mathrm{mg} / \mathrm{mL}$ ) from the sphere-on-Si geometry (Figure 1). (a, b) PS-112K/PMMA-133K at the weight ratio of 3/7 (a) before and (b) after removing PS with cyclohexane. The rings were at the intermediate region, $X_{2}$. (c, d) PS-112K/PMMA-534K at the weight ratio of 3/7 (c) before and (d) after removal of PS with cyclohexane. The rings were in between the intermediate region, $X_{2}$, and the innermost region, $X_{3}$. The arrow indicates the movement of solution front toward the center of the sphere/Si contact. Scale bar $=70 \mu \mathrm{m}$ in all images.

arrows in Figure 3d,h,l as well as seen in Figure 2b). This observation suggests that given the PS/PMMA weight ratio of 3/7, a part of PS was deposited in the middle of the PMMA matrix in the PS/PMMA ring. The depth, $d$, and the width, $\Delta$, of the trench decreased from $d=145 \mathrm{~nm}$ and $\Delta=4 \mu \mathrm{m}$ at $X_{1}$ to $100 \mathrm{~nm}$ and $2.5 \mu \mathrm{m}$ at $X_{2}$ to $80 \mathrm{~nm}$ and $2 \mu \mathrm{m}$ at $X_{3}$ as the solution front moved inward. The data also suggest a preferential segregation of PMMA on the Si substrate in the rings that would otherwise form fingering instabilities, as seen when PS solely used as the nonvolatile solute, ${ }^{17,18}$ and be washed away with cyclohexane. Close examination of AFM images revealed isolated pit structures residing on the both sides of a ring (large pits occurred near the contact line pinned, i.e., on the right side of each ring, as compared to small pits on the left side of contact line in Figure 3; see a representative inset shown in Figure 3k, which may be due to the coupling of PS domains coalescence with the "stick-slip" motion of the contact line). Taken together, trench-pit structures were yielded in the PS-112K/PMMA-133K blend. The large pits near the contact line had a broad size distribution with the average size being $0.85 \pm 0.35 \mu \mathrm{m}$ at $X_{1}$, $0.7 \pm 0.3 \mu \mathrm{m}$ at $X_{2}$, and $0.6 \pm 0.3 \mu \mathrm{m}$ at $X_{3}$.

For the PS-112K/PMMA-534K blend, $\lambda_{\mathrm{C}-\mathrm{C}}, w$, and $h$ of the stripes progressively decreased from $19 \mu \mathrm{m}, 11 \mu \mathrm{m}$, and 110 $\mathrm{nm}$ at $X_{1}=3525 \mu \mathrm{m}$ (Figure 4a) to $14 \mu \mathrm{m}, 9 \mu \mathrm{m}$, and $95 \mathrm{~nm}$ at $X_{2}=2765 \mu \mathrm{m}$ (Figure $4 \mathrm{e}$ ) to $10 \mu \mathrm{m}, 6 \mu \mathrm{m}$, and $80 \mathrm{~nm}$ at $X_{3}$ $=2010 \mu \mathrm{m}$ (Figure 4i). A randomly distributed pit structures were observed on a ring after removal of PS (Figure 4c,d,g,h,k,l). No trenches were seen. The average sizes of pits were $0.75 \pm$ $0.5 \mu \mathrm{m}$ at $X_{1}, 0.9 \pm 0.6 \mu \mathrm{m}$ at $X_{2}$, and $1.2 \pm 0.6 \mu \mathrm{m}$ at $X_{3}$.

It is worth noting that these self-organized trench-pit and pit structures within the concentric rings resulted from spontaneous phase separation of PS/PMMA and a dewetting process in the late stage of phase segregation, not due to the temperaturegradient-induced Marangoni convection. Since the experiments were conducted in a sealed chamber, the evaporation rate of solvent and the associated capillary flow were readily controlled, and the temperature gradient and the possible convective instabilities were eliminated.

On the basis of the observation that the height profile in between the adjacent rings before and after removal of PS was not flat, as evidenced in Figure 3d,h,l and Figure 4d,h,l, it is clear that a thin PMMA patches $(\sim 15 \mathrm{~nm}$ obtained from AFM measurement on scratched samples, images not shown) was formed in between the adjacent rings on the Si substrate. This is not surprising in light of the fact that a PMMA thin film is stable on a $\mathrm{Si}$ substrate with $2 \mathrm{~nm}$ thick native $\mathrm{SiO}_{x}$ on the surface due to the favorable interfacial interaction between PMMA and the Si substrate (i.e., enthalpic interaction between the carbonyl group of PMMA and the silanol groups on the $\mathrm{Si}$ substrate), ${ }^{24}$ while a PS thin film is unstable owing to an unfavorable interaction between PS and $\mathrm{Si}^{24}$

We now turn our attention to rationalize the formation of kinetically trapped nonequilibrium complex structures in Figures 3 and 4. The lateral and vertical organization of the PS and PMMA domains in the PS/PMMA blend was governed by a complex interplay between preferential segregation of PMMA phase at the Si substrate and phase separation in the ring. The residual solvent played an important role in forming topographic modulations on the ring surface in the late stage of phase segregation. The wetting behavior of liquid A on liquid B can be well described by the spreading coefficient. 
(a)

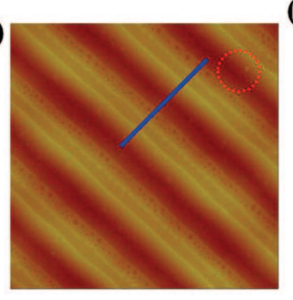

(b)

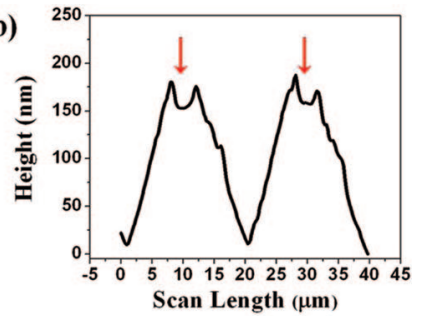

(c)

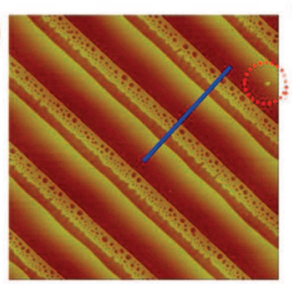

(e)

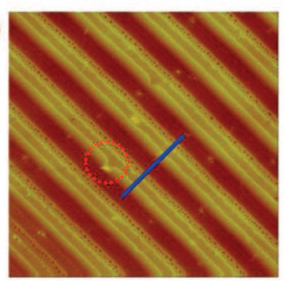

(g)

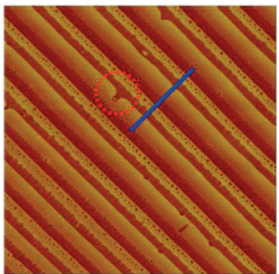

(d)

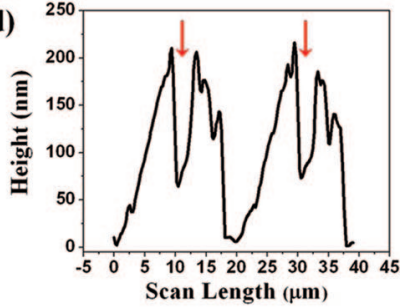

(f)

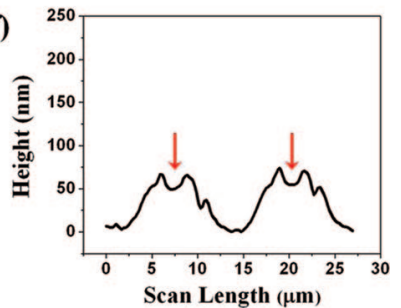

(h)

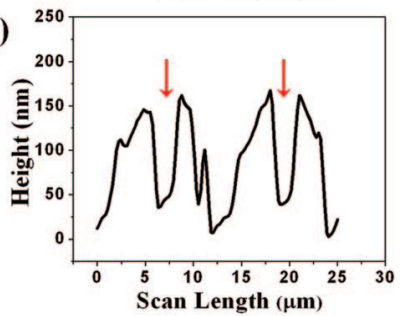

(i)

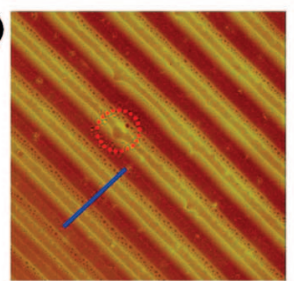

(j)

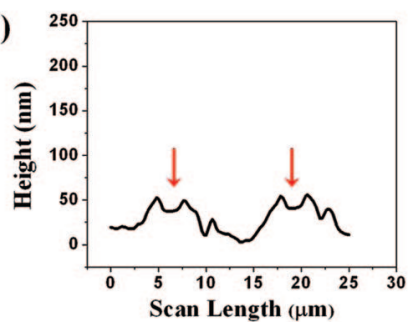

(k)
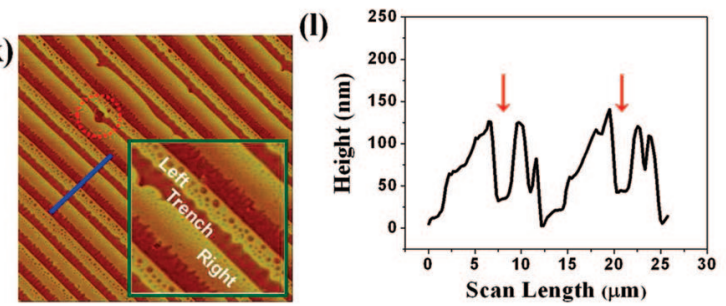

Figure 3. 2D AFM height images and the corresponding profiles over a short scanning distance (blue lines) of concentric rings obtained from drying-mediated self-organization of PS-112K/PMMA-133K toluene solution. As the solution front moved toward the sphere/Si contact center, the center-to-center distance between the adjacent rings, $\lambda_{\mathrm{C}-\mathrm{C}}$, and height of the ring, $h$, decreased progressively. (a-d) Outermost region: $X_{1}(\mathrm{a}, \mathrm{b})$ before and $(\mathrm{c}, \mathrm{d})$ after selective removal of PS. (e-h) Intermediate region: $X_{2}(\mathrm{e}, \mathrm{f})$ before and $(\mathrm{g}, \mathrm{h})$ after removal of PS. $(\mathrm{i}-\mathrm{l})$ Innermost region: $X_{3}(\mathrm{i}, \mathrm{j})$ before and $(\mathrm{k}, \mathrm{l})$ after removal of PS. A closeup in $(\mathrm{k})$ showing the ring composed of a trench in the center and pits on both left and right sides; the image size is $20 \times 20 \mu \mathrm{m}^{2}$. The red circle in the images indicated that the images before and after removal of PS were taken at the same location. The red arrows in (b), (d), (f), (h), (j), and (l) marked the locations of trenches. Scan size is $80 \times 80 \mu \mathrm{m}^{2}$ for all images. (a)


(c)
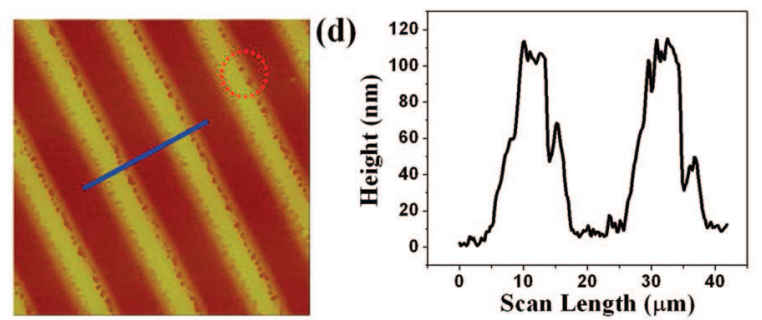

(e)


(g)
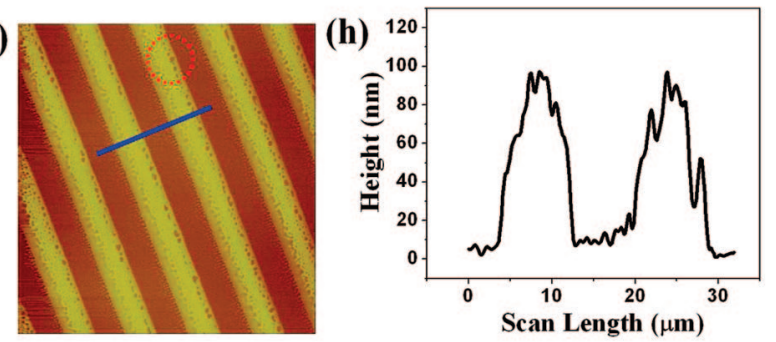

(i)
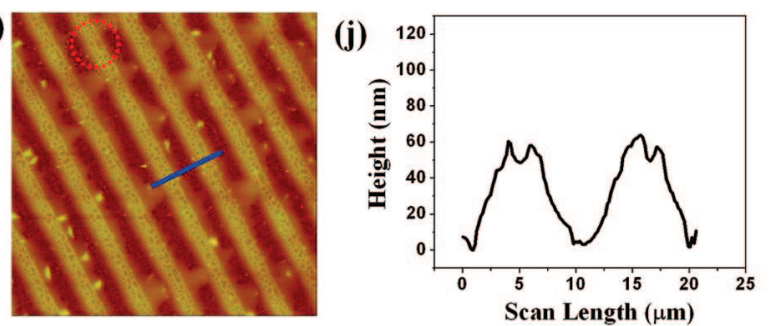

(k)
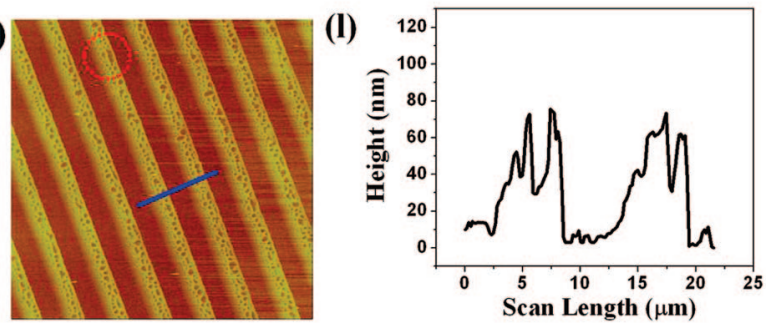

Figure 4. 2D AFM height images and the corresponding profiles over a short scanning distance (blue lines) of concentric rings obtained from drying-mediated self-organization of PS-112K/PMMA-534K toluene solution. As the solution front moved toward the sphere/Si contact center, the center-to-center distance between the adjacent rings, $\lambda_{\mathrm{C}-\mathrm{C}}$, and height of the ring, $h$, decreased progressively. (a-d) Outermost region: $X_{1}(\mathrm{a}, \mathrm{b})$ before and $(\mathrm{c}, \mathrm{d})$ after selective removal of PS. $(\mathrm{e}-\mathrm{h})$ Intermediate region: $X_{2}(\mathrm{e}, \mathrm{f})$ before and $(\mathrm{g}, \mathrm{h})$ after removal of PS. (i-l) Innermost region: $X_{3}(\mathrm{i}, \mathrm{j})$ before and $(\mathrm{k}, \mathrm{l})$ after removal of PS. The red circle in the images indicated that the images before and after removal of PS were taken at the same location. Scan size is $80 \times 80$ $\mu \mathrm{m}^{2}$ for all images. 


$$
S=\gamma_{\mathrm{B}}-\left(\gamma_{\mathrm{A}}+\gamma_{\mathrm{A} / \mathrm{B}}\right)
$$

A completely wets B when $S>0$, while A dewets B when $S$ $<0$ (i.e., partial wetting). ${ }^{36}$ At room temperature, the surface tensions of PS and PMMA are $\gamma_{\mathrm{PS}}=40.7 \mathrm{~mJ} / \mathrm{m}^{2}$ and $\gamma_{\mathrm{PMMA}}=$ $41.1 \mathrm{~mJ} / \mathrm{m}^{2}$, and the interfacial tension between PS and PMMA is $\gamma_{\mathrm{PS} / \mathrm{PMMA}}=3.2 \mathrm{~mJ} / \mathrm{m}^{2}{ }^{37}$ For PS spreading on PMMA, $S_{\text {PS-on-PMMA }}=\gamma_{\text {PMMA }}-\left(\gamma_{\text {PS }}+\gamma_{\text {PS/PMMA }}\right)=-2.8 \mathrm{~mJ} / \mathrm{m}^{2}$. For PMMA spreading on PS, $S_{\text {PMMA-on-PS }}=\gamma_{\mathrm{PS}}-\left(\gamma_{\mathrm{PMMA}}+\right.$ $\left.\gamma_{\mathrm{PS} / \mathrm{PMMA}}\right)=-4.2 \mathrm{~mJ} / \mathrm{m}^{2}$. Therefore, PS and PMMA do not completely wet each other; instead, they dewet. Thus, although PS has lower surface tension than PMMA and is expected to preferentially segregate to the air surface, the formation of a PS/PMMA bilayer in which the PS phase completely resided upon the PMMA phase is not possible due to the negative spreading coefficient, $S$. On the other hand, it has been demonstrated that PS has better solubility in toluene than PMMA. The polymer/solvent interaction parameter, $\chi$, for PS and PMMA in toluene at room temperature are $\chi_{\mathrm{PS}-\text { toluene }}=$ 1.13 and $\chi_{\text {PMMA-toluene }}=1.21 ;^{38}$ thus, a longer time was required to deplete toluene from the PS phase than the PMMA phase. ${ }^{33}$ Therefore, it is possible that residual toluene was trapped in the PS phase after the evaporation-driven self-organization process in which the PMMA phase was already vitrified. Subsequently, the swollen PS chains collapsed upon further evaporation of residual toluene, leading to the PS phase submerged below the surface of the PMMA layer, while not reaching the Si substrate due to unfavorable interfacial interaction between PS and the Si substrate, ${ }^{39}$ as seen in Figure 3a,e,i and Figure 4a,e,i. Taken together, the PS domains formed on the top of the continuous PMMA matrix, possessing the circular shape (i.e., pits in Figures 3 and 4) in order to minimize the interfacial energy between PS and PMMA. Subsequent selective dissolution of PMMA with acetic acid removed the entire hierarchically structured ring patterns, confirming the presence of continuous PMMA matrix on the Si substrate.

The formation of different PS domain structures (i.e., trenchpit structures for the PS-112K/PMMA-133K blend and randomly distributed pits for the PS-112K/PMMA-534K blend) in the PMMA matrix within the PS/PMMA rings may be explained as follows. For the PS-112K/PMMA-133K blend, a PS/PMMA ring was formed directly after solvent evaporation, in which PS and PMMA-133K phase-separated with the residual toluene trapped in the PS phase (i.e., PS pits) (first panel in Figure 5a). For such a ring containing residual toluene, the evaporation flux varied spatially with the highest flux occurred at the edge of the ring. ${ }^{2,40-44}$ Thus, compared to the central region of the ring, the mobility of the PS phase was reduced at the edge, thereby leading to the formation of dispersed small PS domains (i.e., PS pits). Further evaporation of trapped toluene in the PS phase in the central region of the ring led to the PS phase interconnected each other and ultimately coalesced into a trench in the PMMA-133K matrix as illustrated in the second and third panels in Figure 5a. Because of relatively faster depletion of toluene in PMMA than in PS, the PMMA-133K matrix solidified and blocked the pathway of the PS phase that were entrapped to come out to the air surface (gray circles in the lower panel in Figure 5b).

The change of the molecular weight (MW) of PMMA in the blend affected the diffusivity of the system and hence the rate of phase separation. When the MW difference between PS and PMMA became large, sufficient composition fluctuation (i.e., phase separation) cannot proceed easily. The viscosity of the PMMA matrix, $\eta_{\text {PMMA }}$, that is strongly influenced by the MW can be estimated by

$$
\eta_{\mathrm{PMMA}} \sim \mathrm{MW}^{3.4}
$$

(a)

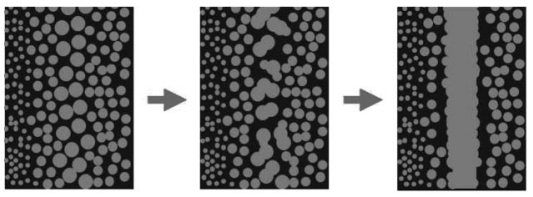

PS-112K/PMMA-133K(3:7)

(b)

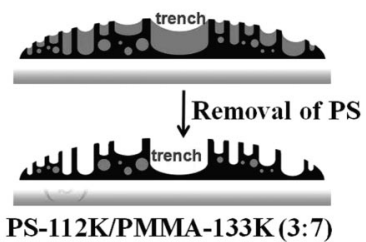

(c)

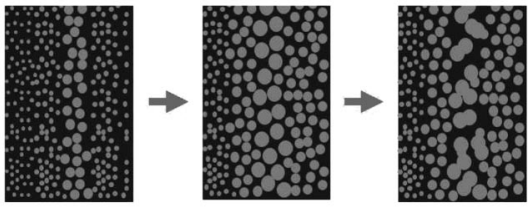

PS-112K/PMMA-534K(3:7)

(d)

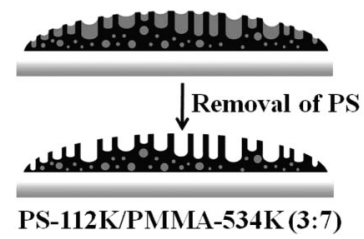

Figure 5. (a, c) Stepwise representation of the formation of the PS (a) trench-pit and (c) pit surface morphologies in the PS-112K/PMMA$133 \mathrm{~K}$ and the PS-112K/PMMA-534K blend, respectively, as residual toluene evaporates (top view). (b, d) Schematic illustration of (b) the trench-pit and (d) the pit structures via the phase separation of PS and PMMA from the PS-112K/PMMA-133K blend and the PS-112K/ PMMA-534K blend, respectively, within the microscopic ring. Upper panels: before removal of PS with cyclohexane. Lower panels: after removal of PS.

The viscosity of PMMA-534K is 65 times larger than that of PMMA-133K $\left(\eta_{\text {PMMA-133K }} \sim(534 / 133)^{3.4} \eta_{\text {PMMA-133K }}=65\right.$ $\left.\eta_{\text {PMMA-133K }}\right)$. This led to a significant reduction in the mobility, $v$, of the PS domains on top of the PMMA-534K matrix ${ }^{24,45,46}$

$$
v \sim\left(\frac{\gamma^{2} \Theta_{\mathrm{e}}}{\eta_{\mathrm{PMMA}}}\right)^{1 / 3}
$$

where $\gamma$ is the effective surface tension, $\gamma=\left[\gamma_{\mathrm{PS}} \gamma_{\mathrm{PMMA}} /\left(\gamma_{\mathrm{PS}}+\right.\right.$ $\left.\left.\gamma_{\text {PMMA }}\right)\right]$, and $\Theta_{\mathrm{e}}$ is the equilibrium contact angle between PS and PMMA, $\cos \Theta_{\mathrm{e}}=\left(\gamma_{\text {PMMA }}-\gamma_{\text {PS/PMMA }}\right) / \gamma_{\text {PS }}$. Thus, the PS domains were not able to efficiently interconnect one another to form a trench in the late stage of phase segregation as seen in Figure 3. Instead, randomly dispersed pit structures were resulted in Figure 5c,d).

\section{Conclusion}

In summary, hierarchically structured polymer blend rings in a gradient concentric ring mode were produced by evaporation of an asymmetric PS/PMMA toluene solution in the sphereon-flat geometry. These complex self-assembled structures were the direct consequence of an interplay between controlled "stick-slip" motion of the contact line, the phase separation of polymer blends, and a dewetting process in the late stage of phase segregation. By changing the MW of the PMMA matrix, the resulting concentric rings exhibited different surface morphologies. At relatively low MW of PMMA (PMMA-133K), trench-pit structures were formed in the PS-112K/PMMA-133K 
blend. At high molecular weight of PMMA (PMMA-534K), pit structures were observed in the PS-112K/PMMA-534K blend. These gradient, hierarchically structured polymer blends rings may serve as a platform to study cell motility and adhesion, the confinement of transmembrane cell receptors, neuron guidance, and other biological processes. ${ }^{47,48}$

Acknowledgment. We gratefully acknowledge support from the National Science Foundation (NSF CAREER Award CBET0844084) and the Key Laboratory of Molecular Engineering of Polymers at Fudan University (Ministry of Education, China).

Supporting Information Available: Optical micrographs of the PS-112K/PMMA-133K at the weight ratio of PS- $112 \mathrm{~K}$ to PMMA-133K $=7 / 3$ and $5 / 5$ formed at the different regions. This material is available free of charge via the Internet at http:// pubs.acs.org.

\section{References and Notes}

(1) Adachi, E.; Dimitrov, A. S.; Nagayama, K. Langmuir 1995, 11, 1057.

(2) Deegan, R. D.; Bakajin, O.; Dupont, T. F.; Huber, G.; Nagel, S. R.; Witten, T. A. Nature (London) 1997, 389, 827.

(3) Karthaus, O.; Grasjo, L.; Maruyama, N.; Shimomura, M. Chaos 1999, $9,308$.

(4) Nguyen, V. X.; Stebe, K. J. Phys. Rev. Lett. 2002, 88, 164501.

(5) Martin, C. P.; Blunt, M. O.; Pauliac-Vaujour, E.; Stannard, A.; Moriarty, P. Phys. Rev. Lett. 2007, 99, 116103.

(6) Pauliac-Vaujour, E.; Stannard, A.; Martin, C. P.; Blunt, M. O.; Notingher, I.; Moriarty, P. J.; Vancea, I.; Thiele, U. Phys. Rev. Lett. 2008, 100, 176102 .

(7) Thiele, U.; Mertig, M.; Pompe, W. Phys. Rev. Lett. 1998, 80, 2869

(8) Huang, J.; Fan, R.; Connor, S.; Yang, P. D. Angew. Chem., Int. Ed. 2007, 46, 2414

(9) Khanal, B. P.; Zubarev, E. R. Angew. Chem., Int. Ed. 2007, 46, 2195.

(10) Bigioni, T. P.; Lin, X. M.; Nguyen, T. T.; Corwin, E. I.; Witten, T. A.; Jaeger, H. M. Nat. Mater. 2006, 5, 265.

(11) Huang, J.; Kim, F.; Tao, A. R.; Connor, S.; Yang, P. D. Nat. Mater. $\mathbf{2 0 0 5}, 4,896$.

(12) Yabu, H.; Shimomura, M. Adv. Funct. Mater. 2005, 15, 575.

(13) Hong, S. W.; Xu, J.; Xia, J.; Lin, Z. Q.; Qiu, F.; Yang, Y. L. Chem. Mater. 2005, 17, 6223.

(14) Xu, J.; Xia, J.; Hong, S. W.; Lin, Z. Q.; Qiu, F.; Yang, Y. L. Phys. Rev. Lett. 2006, 96, 066104.

(15) Hong, S. W.; Giri, S.; Lin, V. S. Y.; Lin, Z. Q. Chem. Mater. 2006 18,5164 .

(16) Hong, S. W.; Xu, J.; Lin, Z. Q. Nano Lett. 2006, 6, 2949.
(17) Hong, S. W.; Xia, J.; Byun, M.; Zou, Q.; Lin, Z. Q. Macromolecules 2007, 40, 2831.

(18) Hong, S. W.; Xia, J.; Lin, Z. Q. Adv. Mater. 2007, 19, 1413.

(19) Xu, J.; Xia, J.; Lin, Z. Q. Angew. Chem., Int. Ed. 2007, 46, 1860.

(20) Wang, J.; Xia, J.; Hong, S. W.; Qiu, F.; Yang, Y.; Lin, Z. Q. Langmuir 2007, 23, 7411.

(21) Byun, M.; Hong, S. W.; Zhu, L.; Lin, Z. Q. Langmuir 2008, 24, 3525.

(22) Hong, S. W.; Jeong, W.; Ko, H.; Kessler, M. R.; Tsukruk, V.; Lin, Z. Q. Adv. Funct. Mater. 2008, 18, 2114.

(23) Jones, R. A. L.; Norton, L. J.; Kramer, E. J.; Bates, F. S.; Wiltzius, P. Phys. Rev. Lett. 1991, 66, 1326.

(24) Lambooy, P.; Phelan, K. C.; Haugg, O.; Krausch, G. Phys. Rev. Lett. 1996, 76, 1110.

(25) Steiner, U.; Klein, J.; Eiser, E.; Budkowski, A.; Fetters, L. J. Science 1992, 258, 1126.

(26) Wang, H.; Composto, R. J. Macromolecules 2002, 35, 2799.

(27) Thiele, U.; Madruga, S.; Frastia, L. Phys. Fluids 2007, 19, 122106.

(28) Clarke, N. Eur. Phys. J. E 2004, 14, 207.

(29) Clarke, N. Macromolecules 2005, 38, 6775.

(30) de Gennes, P. G. Rev. Mod. Phys. 1985, 57, 827.

(31) Geoghegan, M.; Krausch, G. Prog. Polym. Sci. 2003, 28, 261.

(32) Walheim, S.; Schaffer, E.; Mlynek, J.; Steiner, U. Science 1999, 283, 520 .

(33) Walheim, S.; Boltau, M.; Mlynek, J.; Krausch, G.; Steiner, U. Macromolecules 1997, 30, 4995.

(34) Böltau, M.; Walheim, S.; Mlynek, J.; Krausch, G.; Steiner, U. Nature (London) 1998, 391, 877.

(35) Muller-Buschbaum, P.; Bauer, E.; Pfister, S.; Roth, S. V.; Burghammer, M.; Riekel, C.; David, C.; Thiele, U. Europhys. Lett. 2006, 73, 35.

(36) Young, T. Trans. R. Soc. London 1805, 5, 65.

(37) Wu, S. J. Phys. Chem. 1970, 74, 632.

(38) Mark, J. E. Physical Properties of Polymers Handbook; 2007; Chapter $16, \mathrm{p} 289$.

(39) Lin, Z.; Kerle, T.; Russell, T. P.; Schaffer, E.; Steiner, U. Macromolecules 2002, 35, 6255-6262.

(40) Deegan, R. D. Phys. Rev. E 2000, 61, 475.

(41) Deegan, R. D.; Bakajin, O.; Dupont, T. F.; Huber, G.; Nagel, S. R.; Witten, T. A. Phys. Rev. E 2000, 62, 756.

(42) Rabani, E.; Reichman, D. R.; Geissler, P. L.; Brus, L. E. Nature (London) 2003, 426, 271.

(43) Tirumkudulu, M. S.; Russel, W. B. Langmuir 2004, 20, 2947.

(44) Hu, H.; Larson, R. G. J. Phys. Chem. B 2002, 106, 1334.

(45) Brochard-Wyart, F.; Martin, P.; Redon, C. Langmuir 1993, 9, 3682.

(46) Redon, C.; Brochard-Wyart, F.; Rondelez, F. Phys. Rev. Lett. 1991, 66, 715.

(47) Purrucker, O.; Fortig, A.; Ludtke, K.; Jordan, R.; Tanaka, M. J. Am. Chem. Soc. 2005, 127, 1258.

(48) Chen, X.; Hirtz, M.; Fuchs, H.; Chi, L. Langmuir 2007, 23, 2280.

\section{MA801864N}

